\title{
ATIVIDADE FÍSICA COMO DISCRIMINADOR DA AUSÊNCIA DE HIPERTENSÃO ARTERIAL EM HOMENS ADULTOS
}

\author{
PHYSICAL ACTIVITY AS DISCRIMINATOR OF THE ABSENCE OF HYPERTENSION IN ADULT MEN
}

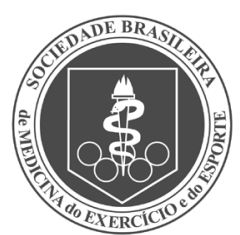

Artigo Original

\author{
ACTIVIDAD FÍSICA COMO DISCRIMINADOR DE LA AUSENCIA DE HIPERTENSIÓN ARTERIAL \\ EN HOMBRES ADULTOS
}

\begin{abstract}
Francisco Jose Gondim Pitanga' (Profissional de Educação Física)

Luiz Alberto Bastos de Almeida² (Profissional de Educação Física) Marcela Mota Freitas ${ }^{3}$ (Profissional de Educação Física) Cristiano Penas Seara Pitanga ${ }^{4}$ (Profissional de Educação Física)

Carmem Cristina Beck

(Profissional de Educação Física)
\end{abstract}

1. Faculdade de Educação da Universidade Federal da Bahia (UFBA), Salvador, BA, Brasil.

2. Universidade Estadual de Feira de Santana(UEFS), Núcleo de Estudos e Pesquisa em Atividade Física e Saúde (NEPAFS), Feira de Santana, BA, Brasil.

3. Instituto Federal da Bahia (IFBA), Salvador, BA, Brasil.

4. Centro Universitário Jorge Amado (UNIJORGE) e Laboratório Baiano de Ciencias do Esporte (SPORTCLIN),

Salvador, BA-Brasil.

5.Instituto Federal de Santa Catarina (IFSC), Florianópolis, SC, Brasil.

\section{Correspondência:}

Luiz Alberto Bastos de Almeida, Departamento de Saúde, Universidade Estadual de Feira de Santana, Av. Transnordestina, s/n, Novo Horizonte, 44036-900, Feira de Santana, BA, Brasil.

lulalong1000@yahoo.com.br

\section{RESUMO}

Introdução: Diversos estudos epidemiológicos demonstram que a atividade física reduz a pressão arterial, porém a quantidade necessária para que estes benefícios sejam alcançados permanece especulativa. Objetivo: Analisar a quantidade (duração e intensidade) de atividade física em seus diferentes domínios (trabalho, deslocamento, atividade doméstica e tempo livre) como discriminador da ausência de hipertensão arterial em adultos do sexo masculino. Métodos: Estudo transversal realizado na cidade de Lauro de Freitas, Bahia, Brasil com amostra constituída por 220 indivíduos do sexo masculino com idade superior a 18 anos. Foram construídas diversas curvas Receiver Operating Characteristic (ROC) e comparadas às áreas sobre as mesmas entre a quantidade de atividade física em seus diferentes domínios e a ausência de hipertensão arterial. Verificou-se também a sensibilidade e especificidade para a quantidade de atividade física necessária para prevenção de hipertensão arterial. Foi utilizado o intervalo de confiança a 95\%. Resultados: Entre os diferentes domínios de atividade física analisados encontrou-se maior significância estatística nas áreas sob a curva ROC para a atividade física no trabalho e no tempo livre. A caminhada não foi boa discriminadora para a ausência de hipertensão arterial em nenhum dos domínios analisados. Observou-se também que $180 \mathrm{~min} / \mathrm{semana}$ de atividade física moderada, acumulada nos diferentes domínios foi o melhor ponto de corte para discriminar a ausência de hipertensão arterial. Conclusão: A prática da atividade física, principalmente de intensidade moderada, com duração de pelo menos $180 \mathrm{~min} / \mathrm{semana}$ deve ser sugerida para indivíduos do sexo masculino visando contribuir para a prevenção da hipertensão arterial.

Palavras-chave: atividade física, hipertensão, curva ROC, prevenção de doenças.

\section{ABSTRACT}

Introduction: Several epidemiological studies show that physical activity reduces blood pressure but the amount needed to bring these benefits remains speculative. Objective: To analyze the amount (duration and intensity) of physical activity in its different domains (work, commuting, household activity and leisure time) as discriminator of the absence of hypertension in adult males. Methods: Cross-sectional study conducted in the city of Lauro de Freitas, Bahia, Brazil with sample of 220 males aged over 18 years. Several Receiver Operating Characteristic $(R O C)$ curves were constructed which were compared to the areas between the amount of physical activity in their various domains and the absence of hypertension. For the amount of physical activity needed to prevent hypertension, sensitivity and specificity were also checked. The confidence interval of 95\% was used. Results: Among the different domains of physical activity analyzed we found a greater statistical significance in the areas under the ROC curve for physical activity at work and leisure time. Hiking was not a good discriminator for the absence of hypertension in any of the domains analyzed. It was also observed that 180 minutes/week of moderate physical activity accumulated in the different domains was the best cutoff point for discriminating the absence of hypertension. Conclusion: The practice of physical activity, especially moderate intensity, lasting at least 180 minutes/week should be suggested for males to contribute to the prevention of hypertension.

Keywords: physical activity, hypertension, ROC curve, disease prevention.

\section{RESUMEN}

Introducción: Diversos estudios epidemiológicos demuestran que la actividad física reduce la presión arterial, aunque la cantidad necesaria para que estos beneficios sean alcanzados permanece especulativa. Objetivo: Analizar la cantidad (duración e intensidad) de actividad física en sus diferentes dominios (trabajo, desplazamiento, actividad doméstica y tiempo libre) como discriminador de la ausencia de hipertensión arterial en adultos del sexo masculino. Métodos: Estudio transversal realizado en la ciudad de Lauro de Freitas, Estado de Bahia, Brasil, con muestra constituida por 220 individuos del sexo masculino con edad superior a 18 años. Fueron construidas diversas curvas Receiver Operating Characteristic (ROC) y comparadas a las áreas sobre las mismas entre la cantidad de actividad física en sus diferentes dominios y la ausencia de hipertensión arterial. Se verificó también la sensibilidad y especificidad para la cantidad de actividad física necesaria para prevención de hipertensión arterial. Fue utilizado el intervalo de confianza a 95\%. Resultados: Entre los diferentes dominios de actividad física analizados se encontró mayor significado estadístico en las áreas bajo la curva ROC para la actividad física en el trabajo y en el tiempo libre. La caminata no fue buena discriminadora para la ausencia de hipertensión arterial en ninguno de los dominios 
analizados. Se observó también que $180 \mathrm{~min} / \mathrm{semana}$ de actividad física moderada, acumulada en los diferentes dominios fue el mejor punto de corte para discriminar la ausencia de hipertensión arterial. Conclusión: La práctica de la actividad física, principalmente de intensidad moderada, con duración de por los menos $180 \mathrm{~min} / \mathrm{semana}$ debe ser sugerida para individuos del sexo masculino buscando contribuir para la prevención de la hipertensión arterial.

Palabras clave: actividad física, hipertensión, curva ROC, prevención de enfermedades.

\section{INTRODUÇÃO}

A atividade física (AF) compreendida como fenômeno com componentes e determinantes de ordem social, cultural, comportamental, ambiental e biológica, é definida como qualquer movimento corporal provocado pela musculatura esquelética que resulte em gasto energético acima dos níveis de repouso e pode ser representada por atividades domésticas, atividades no trabalho, atividades no tempo livre e atividades de deslocamento'.

A hipertensão arterial (HA) é encontrada em níveis epidêmicos em adultos das sociedades industrializadas, favorecendo o aumento do risco para desenvolvimento de diversas patologias cardiovasculares ${ }^{2}$.

Numerosas evidências de estudos epidemiológicos demonstram que a AF pode estar inversamente associada a $\mathrm{HA}_{1}^{3-6}$ porém,a quantidade necessária para alcançar estes benefícios permanece especulativa.

Recentes publicações ${ }^{7-10}$ têm procurado identificar a quantidade de AF necessária para a prevenção do diabetes, excesso de gordura visceral e comorbidades cardiovasculares. Os resultados vêm demonstrando que a AF, principalmente na intensidade moderada, com duração variando entre 150 e 285 min por semana ou com gasto calórico entre 1.601 e 2.283 kcal/semana parece ser boa estratégia de prevenção. No entanto, dois destes estudos demonstraram que para indivíduos do sexo masculino a caminhada isoladamente parecer não ser uma boa opção para prevenção de diabetes ${ }^{7,8}$. Assim, tornam-se necessários trabalhos que também tentem identificar a quantidade de AF suficiente para proteção da HA, principalmente em homens.

Os mecanismos pelos quais a AF reduz os níveis de PA permanecem especulativos. Conforme o American College of Sports Medicine (ACSM) ${ }^{2}$ as principais evidências apontam: (a) diminuição dos níveis de insulina, com consequente redução da retenção do sódio renal e tônus simpático basal; (b) redução dos níveis das catecolaminas; e (c) liberação, pela musculatura esquelética, de substâncias vasodilatadoras na circulação.

Assim, a determinação da quantidade de AF necessária ou suficiente para a prevenção da HA pode otimizar os programas de práticas corporais, fazendo com que os benefícios da prática regular de AF possam ser maximizados.

Desta forma, o objetivo do estudo foi analisar a quantidade (duração e intensidade) de atividade física em seus diferentes domínios (trabalho, deslocamento, atividade doméstica e tempo livre) para discriminar a ausência de hipertensão arterial em adultos do sexo masculino.

\section{MÉTODOS}

Trata-se de estudo de corte transversal realizado no município de Lauro de Freitas, situado no nordeste do estado da Bahia, Brasil, parte da Região Metropolitana de Salvador, com extensão territorial de 59 quilômetros quadrados. O município de Lauro de Freitas possui IDH de 0,771 , PIB per capita de $\mathrm{R} \$ 12.046,00$ e população estimada de 138.240 habitantes ${ }^{11}$

O calculo da amostra foi baseado em Kisch ${ }^{12}$ levando-se em consideração os seguintes parâmetros: tamanho da população de 138.240 habitantes, prevalência de atividade física de 50\% (maiorprevalência entre as variáveis analisados no estudo),tendo como base estudo realizado no estado de São Paulo, Brasil ${ }^{13}$, nível de confiança de 95\% de precisão, erro de cinco pontos percentuais. A amostra foi calculada em 500 indivíduos de ambos os sexos com acréscimo de 20\%, totalizando 600 adultos com idade igual ou maior que 18 anos.

O acréscimo de 100 indivíduos na amostra mínima calculada previu a exclusão das residências desabitadas, moradores ausentes, moradores inelegíveis, pessoas acamadas por motivo de doença, pessoas que se recusaram a responder o questionário.

A amostra foi probabilística, em múltiplos estágios e por conglomerados de classes sociais informados pela Secretaria de Ação Social da Prefeitura da cidade de Lauro de Freitas, à partir do poder aquisitivo dos moradores dos bairros. Considerou-se como classe A (alta e média alta), classe B (média), classe C (média e baixa) e classe D (baixa e pobreza).

Inicialmente o mapa da cidade foi dividido em microrregiões de acordo com a classe social predominante. Em seguida foram sorteadas 25 ruas da cidade de Lauro de Freitas pertencentes aos quatro níveis sociais (Classe A, B, C, D). A divisão das ruas foi proporcional ao nível socioeconômico e obedeceu ao seguinte quantitativo: seis ruas em cada um dos conglomerados A, B e C e sete ruas na classe D. Em cada rua, treze domicílios foram sorteados por amostra sistemática. O intervalo entre as casas variou de acordo com a quantidade de domicílios encontrados em cada rua. Em cada residência visitada foram sorteados dois indivíduos adultos (um homem e uma mulher), respeitando a proporcionalidade da distribuição dos sexos na população.

Desta forma, ao final da coleta de dados a amostra ficou constituída de 522 pessoas, 220 do sexo masculino e 302 do sexo feminino. Para efeito do presente estudo foram utilizados apenas os indivíduos do sexo masculino totalizando 220 pessoas.

O presente estudo foi aprovado pelo Comitê de Ética da Faculdade Adventista de Fisioterapia (FAFIS) localizada na cidade de Cachoeira, Bahia, Brasil, conforme parecer nº. 0033/2007. Todos os participantes da pesquisa assinaram Termo de Participação Livre e Esclarecida e foram entrevistados em domicílio.

\section{Coleta de dados}

A coleta de dados aconteceu de março de 2007 a abril de 2008. Cinco avaliadores foram devidamente preparados e treinados para todas as etapas do trabalho. $O$ índice de confiabilidade interavaliadores foi testado para aplicação do IPAQ por meio do coeficiente Kappa, o qual apresentou bom índice de concordância $(0.61)^{14}$. O erro técnico de medidas para o peso e a estatura foi considerado baixo $(1.2 \%)^{15}$.

Os dados demográficos e as variáveis relacionadas à saúde foram coletados por meio de questionário. Para obtenção dos dados antropométricos utilizou-se os seguintes protocolos: a estatura foi mensurada com uma trena antropométrica em aço Sanny (Brasil), com os indivíduos descalços, em posição ereta, com pés e calcanhares unidos e encostados na parede, braços estendidos ao longo do corpo, respiração normal seguindo o Plano de Frankfurt ${ }^{16}$.

O peso foi mensurado duas vezes utilizando-se balanças da marca Plenna com precisão de 100 gramas, todas aferidas previamente pelo Instituto Nacional de Metrologia (INMETRO). Ao entrevistado era 
solicitado que subisse na balança com os pés descalços, com o mínimo de roupa possível ${ }^{16}$.

Para a medida de atividade física foi utilizado o International Physical Activity Questionnaire (IPAQ) versão longa, constituído de questões acerca da frequência e duração das atividades físicas (caminhada, moderada e vigorosa) realizadas nos quatro domínios (trabalho, deslocamento, atividades domésticas e no tempo livre) ${ }^{17}$. Os valores de atividade física foram relatados em min/semana por meio da multiplicação da frequência semanal pela duração de cada uma das atividades realizadas.

A presença ou ausência de hipertensão foiauto-referida considerando-se o diagnóstico médico prévio determinado por meio de pergunta traduzida para o português e adaptada do NHANES III: "Algum médico ou profissional de saúde já disse que você tinha pressão alta ou hipertensão arterial?" Tal metodologia foi validada no Brasil por Lima-Costa et al. em 2004 (Projeto Bambuí) ${ }^{18}$.

Caso o indivíduo não soubesse informar, era realizada uma apreciação da medicação prescrita pelo médico e verificada a presença de anti-hipertensivos orais entre os medicamentos.

\section{Análise estatística}

O poder discriminatório e os pontos de corte dos diferentes domínios da atividade física para ausência de hipertensão arterial foram identificados por meio das curvas Receiver Operating Characteristic (ROC), frequentemente utilizadas para determinação de pontos de corte em testes diagnósticos ou de triagem ${ }^{19}$.

Inicialmente foi identificada a área total sob a curva ROC entre a atividade física (caminhada, moderada e vigorosa) em seus diferentes domínios (trabalho, deslocamento, atividade doméstica, tempo livre) e a ausência de hipertensão arterial. Quanto maior área sob a curva ROC, maior o poder discriminatório dos diferentes domínios de atividade física para ausência de hipertensão arterial. Utilizou-se intervalo de confiança (IC) a 95\%. O cálculo do IC a 95\% determina se a capacidade preditiva dos indicadores de hipertensão arterial não é devido ao acaso, não devendo o seu limite inferior ser menor do que $0,50^{20}$.

Na sequência foram encontrados pontos de corte em min/semana da atividade física para ausência de hipertensão arterial, com suas respectivas sensibilidade e a especificidade. Os pontos de corte foram identificados segundo o mais adequado equilíbrio entre a sensibilidade e a especificidade das variáveis intensidade (caminhada, moderada e vigorosa) e duração (min/semana) da atividade física como discriminadoras da ausência de hipertensão arterial. Os dados foram analisados através do programa estatístico "STATA", versão 7.0.

\section{RESULTADOS}

As características da amostra estão na demonstradas na tabela 1. Observa-se que os homens são mais ativos na atividade física realizada no trabalho, seguido da atividade física doméstica e tempo livre. 0 domínio onde os mesmos são menos ativos é o da atividade física no deslocamento.

Na tabela 2 pode-se observar as áreas sob as curvas ROC com seus respectivos intervalos de confiança da quantidade de atividade física (duração e intensidade) em seus diferentes domínios como preditores da ausência da hipertensão arterial. De modo geral, as maiores áreas foram observadas nos domínios da atividade física de tempo livre e no trabalho. Observou-se também que as áreas sob a curva ROC foram mais significativas nas intensidades moderada e vigorosa. Na caminhada as áreas sob as curvas ROC não foram significativas em nenhum dos domínios analisados.

Na tabela 3 observam-se as áreas sob as curvas ROC, com seus respectivos intervalos de confiança, entre a quantidade de atividade
Tabela 1. Características dos participantes de acordo com as variáveis analisadas no estudo.

\begin{tabular}{|c|c|}
\hline Variáveis & Masculino $(n=220)$ \\
\hline Idade (anos) & $40,2 \pm 15,3(18,0-85,0)$ \\
\hline$(\mathrm{kg})$ & $75,0 \pm 14,07(41,0-126,0)$ \\
\hline Estatura (m) & $1,70 \pm 0,081(1,41-1,92)$ \\
\hline \multicolumn{2}{|c|}{ Atividade física trabalho (min/semana) } \\
\hline Caminhada & $208,2 \pm 487,7(0-2880,0)$ \\
\hline Moderada & $142,8 \pm 448,6(0-2940,0)$ \\
\hline Vigorosa & $44,2 \pm 156,6(0-1080,0)$ \\
\hline Total & $395,5 \pm 857,8(0-5310,0)$ \\
\hline \multicolumn{2}{|c|}{ Atividade física no deslocamento (min/semana) } \\
\hline Caminhada & $99,4 \pm 189,1(0-1440,0)$ \\
\hline Bicicleta & $52,8 \pm 126,6(0-840,0)$ \\
\hline Total & $152,2 \pm 239,1(0-1620,0)$ \\
\hline \multicolumn{2}{|c|}{ Atividade física doméstica (min/semana) } \\
\hline Moderada em casa & $105,8 \pm 277,6(0-1890,0)$ \\
\hline Moderada no quintal & $112,4 \pm 300,4(0-2400,0)$ \\
\hline Vigorosa no quintal & $39,2 \pm 171,0(0-1680,0)$ \\
\hline Total & $257,4 \pm 514,3(0-4200,0)$ \\
\hline \multicolumn{2}{|c|}{ Atividade física no tempo livre (min/semana) } \\
\hline Caminhada & $46,5 \pm 114,9(0-700,0)$ \\
\hline Moderada & $84,2 \pm 215,5(0-1680,0)$ \\
\hline Vigorosa & $31,0 \pm 95,3(0-720,0)$ \\
\hline Total & $161,7 \pm 285,1(0-1710,0)$ \\
\hline \multicolumn{2}{|c|}{ Atividade física total ${ }^{\S}(\mathrm{min} / \mathrm{semana})$} \\
\hline Caminhada & $354,1 \pm 545,2(0-2940,0)$ \\
\hline Moderada & $497,9 \pm 711,2(0-4535,0)$ \\
\hline Vigorosa & $114,7 \pm 242,8(0-1680,0)$ \\
\hline Total & $966,8 \pm 1105,5(0-7250,0)$ \\
\hline \multicolumn{2}{|l|}{ Hipertensão arterial (\%) } \\
\hline Presença & 17,7 \\
\hline Ausência & 82,3 \\
\hline
\end{tabular}

§ Atividade Física Total: quatro domínios (trabalho, deslocamento, doméstica, tempo livre).

Tabela 2. Áreas sob a curva ROC e IC95\% entre a quantidade de atividades física em seus diferentes domínios como discriminador da ausência de hipertensão arterialem adultos do sexo masculino.

\begin{tabular}{|c|c|}
\hline $\begin{array}{l}\text { Quantidade de atividade física em } \\
\text { diferentes domínios }\end{array}$ & Áreas sob a curva ROC \\
\hline \multicolumn{2}{|l|}{ Trabalho } \\
\hline Caminhada & $0,58(0,49-0,66)$ \\
\hline Moderada & $0,58(0,51-0,64) *$ \\
\hline Vigorosa & $0,57(0,54-0,61) *$ \\
\hline Total & $0,62(0,53-0,70) *$ \\
\hline \multicolumn{2}{|l|}{ Tempo livre } \\
\hline Caminhada & $0,45(0,37-0,54)$ \\
\hline Moderada & $0,63(0,57-0,70) *$ \\
\hline Vigorosa & $0,57(0,52-0,62) *$ \\
\hline Total & $0,56(0,46-0,67)$ \\
\hline \multicolumn{2}{|l|}{ Deslocamento } \\
\hline Caminhada & $0,56(0,46-0,66)$ \\
\hline Bicicleta & $0,57(0,49-0,65)$ \\
\hline Total & $0,59(0,48-0,69)$ \\
\hline \multicolumn{2}{|l|}{ Doméstica } \\
\hline Moderada em casa & $0,56(0,47-0,65)$ \\
\hline Moderada no quintal & $0,44(0,34-0,54)$ \\
\hline Vigorosa no quintal & $0,49(0,42-0,56)$ \\
\hline Total & $0,48(0,38-0,58)$ \\
\hline
\end{tabular}

ROC: receiveroperatingcharacteristic; IC95\%:intervalo de confiança a 95\%. *Área sob a curva ROC apresentando poder discriminatório para ausência de hipertensão arterial (Li-IC $\geq 0,50$. 
física acumulada nos diferentes domínios como preditores da ausência da hipertensão arterial. Mais uma vez observa-se que na caminhada as áreas sob as curvas ROC não foram significativas, porém essa significância é confirmada nas atividades realizadas com intensidades moderada e vigorosa.

A tabela 4 e a figura 1 apresentam os pontos de corte, com suas respectivas sensibilidades e especificidades, da quantidade de atividade física acumulada (trabalho, deslocamentos, atividades domésticas e tempo livre) como discriminadores da ausência da hipertensão arterial em adultos do sexo masculino. Constatou-se que $180 \mathrm{~min} / \mathrm{semana}$ de atividade física moderada foi o melhor ponto de corte para predizer a ausência da hipertensão arterial em adultos do sexo masculino.

Tabela 3. Áreas sob a curva ROC e IC95\% entre a quantidade de atividade física acumulada nos diferentes domínios como discriminador da ausência de hipertensão arterialem adultos do sexo masculino.

\begin{tabular}{c|c}
\hline $\begin{array}{c}\text { Atividade física acumulada } \\
\text { (tempo livre, trabalho, deslocamento e doméstica) }\end{array}$ & Áreas sob a curva ROC \\
\hline Caminhada & $0,54(0,44-0,64)$ \\
\hline Moderada & $0,59(0,50-0,70)^{*}$ \\
\hline Vigorosa & $0,61(0,53-0,68)^{*}$ \\
\hline Total & $0,58(0,48-0,68)$ \\
\hline
\end{tabular}

ROC: receiveroperatingcharacteristic; IC95\%:intervalo de confiança a 95\%. *Área sob a curva ROC apresentando poder discriminatório para ausência de hipertensão arterial ( $(\mathrm{i}-\mathrm{IC} \geq 0,50)$.

Tabela 4. Pontos de corte, sensibilidade e especificidade da quantidade de atividade física acumulada nos diferentes domínios como discriminador da ausência de hipertensão arterial em adultos do sexo masculino.

\begin{tabular}{c|c|c|c}
\hline $\begin{array}{c}\text { Padrões da atividade } \\
\text { física total }\end{array}$ & $\begin{array}{c}\text { Ponto de corte } \\
\text { (min/semana) }\end{array}$ & Sensibilidade (\%) & Especificidade (\%) \\
\hline Caminhada & NR & --- & ---- \\
\hline Moderada & 180 & 59,0 & 59,0 \\
\hline Vigorosa & NR & --- & --- \\
\hline Total & NR & ---- & --- \\
\hline
\end{tabular}

NR: indicador não recomendável para a discriminação da ausência de hipertensão arterial (pontos de corte não apresentam boas sensibilidade e especificidade).

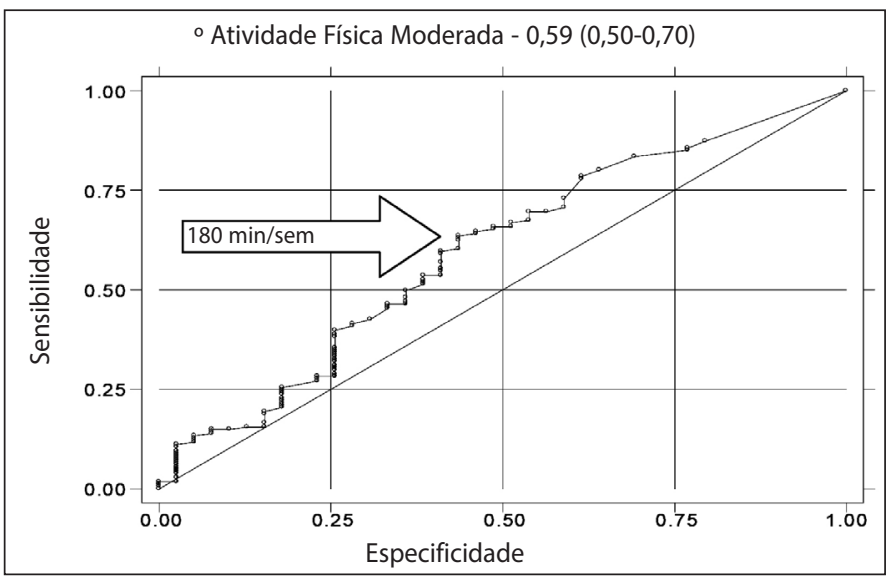

Figura 1. Área sob a curva ROC, com seu respectivo ponto de corte, para atividade física moderada acumulada nos diferentes domínios como discriminador da ausência de hipertensão arterial em adultos do sexo masculino.

\section{DISCUSSÃO}

O estudo demonstra o poder discriminatório da quantidade (duração e intensidade) de atividade física em seus diversos domínios para a ausência de hipertensão arterial em adultos do sexo masculino. Além disso, identifica os pontos de corte, em cada uma das intensidades da atividade física, considerando os valores que apresentaram maior equilíbrio entre sensibilidade e especificidade para discriminar a ausência da hipertensão arterial.
Uma possível limitação do estudo foi a determinação da hipertensão arterial autorreferida, que pode ter provocado subestimação na prevalência desta variável, considerando que muitas pessoas podem desconhecer sua condição de hipertenso. Outra limitação pode ser atribuída à dificuldade na coleta de dados nos bairros de classes $\mathrm{Ce}$ $\mathrm{D}$ por questões de focos de violência. Por outro lado, o instrumento usado para avaliar a duração e intensidade de atividade física (IPAQ), apesar de ser utilizado em diversos estudos nacionais e internacionais pode provocar erros de classificação considerando que os instrumentos do tipo questionário, aplicados na forma de entrevista podem provocar viés de memória. Além disto, este mesmo instrumento, ao classificar a caminhada não considera a intensidade com que a mesma é realizada, fato que pode ter influenciado na não significância desta estratégia para prevenção da hipertensão arterial.

Diversos autores têm demonstrado que a atividade física pode proporcionar benefícios na prevenção e tratamento da hipertensão arteria|3,4,5,6,21. Segundo o ACSM (1993)², indivíduos com hipertensão essencial que praticam atividades físicas regulares tem em média $10 \mathrm{mmHg}$ de redução na pressão arterial sistólica e diastólica melhorando também outros fatores de riscos para as doenças cardiovasculares.

Khan et al., ${ }^{22}$, recomendaram a prática de 30 a 60 min de atividades físicas com uma frequência semanal variando de quatro e sete dias por semana para a prevenção e tratamento da hipertensão arterial. Nota-se que diversos são os estudos que demonstram a importância da atividade física enquanto variável protetora para a hipertensão arterial, ,3,6,22 porém poucos trabalhos tentaram identificar o poder discriminatório de diferentes intensidades e durações de atividades físicas para a ausência de hipertensão arterial em adultos.

No nosso estudo observou-se que a atividade física de tempo livre e no trabalho mostraram-se os melhores discriminadores da ausência de hipertensão arterial em adultos do sexo masculino. Os domínios das atividades físicas domésticas e no deslocamento foram os menos adequados para proteção de hipertensão arterial. Resultados parecidos com relação ao tempo livre e ao trabalho foram observados em recente publicação ${ }^{8}$, a qual sugeriu que a atividade física no lazer foi a mais adequada para prevenção do diabetes.

Outro aspecto que chama atenção no presente estudo é o fato da caminhada não se apresentar como discriminadora da ausência de hipertensão arterial em nenhum dos domínios de atividade física analisados. É importante ressaltar que o instrumento utilizado não analisa a intensidade da caminhada, o que pode ter contribuído para este resultado, já que a caminhada de baixa intensidade provavelmente não provoca alterações metabólicas e cardiovasculares significativas. Concordando com estes resultados, recentes publicações do nosso grupo de pesquisa também demonstraram que a caminhada isoladamente não é uma boa opção para prevenção do diabetes, principalmente em indivíduos do sexo masculino 7,8 .

No presente estudo as atividades moderadas e vigorosas principalmente nos domínios do tempo livre e do trabalho apresentaram-se satisfatórias para discriminar a ausência de hipertensão arterial. Concordando com nossos resultados recente publicação também do nosso grupo de pesquisa demonstrou que atividade física moderada realizada no trabalho ou no tempo livre era boa opção para proteção do diabetes?

Em outro trabalho ${ }^{23}$ que acompanhou durante 30 anos indivíduos do sexo masculino com idade entre 40 e 59 anos demonstrou-se que os sujeitos que praticavam atividades físicas no tempo livre e que tinham maiores níveisde aptidão física apresentaram diminuição substancial na mortalidade por doenças cardiovasculares.

Apesar das evidencias de que adultos que praticam atividades fí- 
sicas pelo menos 150 min semanais em intensidade moderada ou 75 min em intensidade vigorosa passam a obter maiores ganhos a saúde e uma maior proteção para doenças metabólicas e cardiovasculares ${ }^{24}$, no presente estudo observou-se que atividade física moderada realizada por $180 \mathrm{~min} / \mathrm{semana}$ apresentou-se mais eficaz para discriminar a ausência de hipertensão arterial.

Resultados similares foram encontrados em trabalho publicado recentemente ${ }^{7}$, quando foi observado que a atividade física acumulada nas diferentes intensidades (caminhada, moderada e vigorosa) com duração de 185 min por semana foi adequada para proteção de diabetes em homens de etnia negra.

Outro estudo ${ }^{25}$ demonstrou que a combinação de exercícios moderados e vigorosos numa frequência de pelo menos cinco vezes por semana oferecem melhor efeito protetor.

\section{CONCLUSÃO}

Conforme os resultados encontrados no presente trabalho pode-se sugerir que a atividade física de intensidade moderada com duração de pelo menos 180 min/semana parece ser uma boa estratégia para discriminar a ausência, ou seja, para prevenção da hipertensão arterial em adultos do sexo masculino. Sugerem-se novos estudos para tentar encontrar a melhor quantidade de atividade física para a prevenção da hipertensão arterial em mulheres, bem como para a prevenção de outros agravos metabólicos e cardiovasculares em indivíduos de ambos os sexos.

Todos os autores declararam não haver qualquer potencial conflito de interesses referente a este artigo.

\section{REFERÊNCIAS}

1. Caspersen CJ, Powell KE, Christenson G.M. Physical activity, exercise and physical fitness. Public Health Rep. 1985;100:126-31.

2. ACSM - American College of Sports Medicine. Physical activity, physical fitness, and hypertension. Med Sci Sports Exerc. 1993; 25(10): p.i-x.

3. Pitanga FJG, Lessa I. Associação entre Atividade Física no Tempo Livre e Pressão Arterial em adultos. ArqBrasCardiol. 2010;95(4):480-5.

4. Liu L, Kanda T, Sagara M, Hirao S, Yasui N, Negishi H, et al. Leisure-time physicalactivityandotherfactors in relation to blood pressure in Japanese-Americans in Hawaii, USA. Hypertens Res. 2001; 24 (2):145-51.

5. Sofi F, Capalbo A, Marcucci R, Gori AM, Fedi S, Macchi C, et al. Leisure time but not occupational physical activity significantly affects cardiovascular risk factors in an adult population. Eur J Clin Invest. 2007; 37 (12):947-53.

6. Sohn AJ, Hasnain M, Sinacore JM. Impact of exercise (walking) on blood pressure levels in African American adults with newly diagnosed hypertension. EthnDis. 2007;17(3):503-7.

7. Pitanga FJG, Lessa I, Barbosa, PJB, Barbosa SJO, Costa MC, Lopes AS. Atividade física na prevenção do diabetes em etnia negra: quanto é necessário? RevAssocMed Bras. 2010;56(6):697-704.

8. Pitanga FJG, Almeida LAB., Freitas MM, Pitanga CPS, Beck CC. Padrões de atividade física em diferentes domínios e ausência de diabetes em adultos. Motri.2010;6(1):5-17.

9. Pitanga FJG, Lessa I, Pitanga CPS, Costa MC. Atividade física na prevenção de co-morbidades cardiovasculares em mulheres obesas: Quanto é necessário? Rev Bras AtivFísSaúde. 2011;16(4):334-8.

10. Pitanga CP, Pitanga FJ, Beck CC, Gabriel RE, Moreira MH. Level of physical activity in the prevention of excess visceral fat in postmenopausal women: how much is needed? ArqBrasEndocrinolMetabol. 2012;56(6):358-63.

11. DATASUS. Departamento de Informática do SUS. Informações em Saúde, demográficas e socioeconômicas: população residente Estimativas para o TCU-Bahia 2005. Disponível em http://tabnet.datasus. gov.br/cgi/tabcgi.exe?ibge/cnv/poptBA.def [Acessado em 19 de março de 2010].

12. Kish L. Survey Sampling. New York: John Wiley and Sons; 1965.

13. Matsudo SM, Matsudo VR, Araújo T, Andrade D, Andrade E, Oliveira LC, et al. Nível de atividade física da população do estado de São Paulo: Análise de acordo com o gênero, idade e nível sócio-econômico, distribuição geográfica e de conhecimento. RevBrasCiên e Mov. 2002;10(4):41-50.

14. Pereira MG. Epidemiologia: teoria e prática. Rio de Janeiro: Guanabara Koogan; 1995.

15. Gore C, Norton K, Olds T, Whittin-gham N, Birchall K, Clough M, et al. Erros de medição em antropometria. In Norton K \&Olds T (Eds.), Antropométrica. Porto Alegre: Artmed, 2005. p. 375-91.

16. Pitanga FJG. Teste, medidas e avaliação em educação física. 4aed. São Paulo: Editora Phorte; 2007.

17. Matsudo S, Timóteo A, Matsudo V, Andrade D, Andrade E, Oliveira C, et al. Questionário internacional de atividade física (IPAQ): Estudo de validade e reprodutibilidade no Brasil. Rev Bras AtivFísSaúde. 2001;(6):5-18

18. Lima-Costa MF, Peixoto SVE, Firmo JOA, Validade da hipertensão arterial auto-referida e seus determinantes (projeto Bambuí) Rev. Saúde Pública. 2004;38 (5):637-42.

19. Erdreich LS, Lee ET. Use of relative operating characteristics analysis in epidemiology: a method for dealing with subjective judgment. Am J Epidemiol. 1981;114(5):649-62.

20. Schisterman EF, Faraggi D, Reiser B, Trevisan M. Statistical inference for the area under the receiver operating characteristic curve in the presence of random measurement error. Am J Epidemiol. 2001;154(2):174-9.

21. Bryan SN, Katzmarzyk PT. The association between meeting physical activity guidelines and chronic diseases among Canadian adults. J PhysActHealth. 2011;8(1):10-7.

22. Khan NA, McAlister FA, Rabkin SW, Padwal R, Feldman RD, Campbell NR, et al. Canadian Hypertension Education Program. Can J Cardiol. 2006;22(7):583-93.

23. Holtermann A, Mortensen OS, Burr H, Søgaard K, Gyntelberg F, Suadicani P. Fitness, work, and leisure-time physical activity and ischaemic heart disease and all-cause mortality among men with pre-existing cardiovascular disease. Scand J Work Environ Health. 2010; 36(5):349-55

24. O'Donovan G, Blazevich AJ, Boreham C, Cooper AR, Crank H, Ekelund U, et al. The ABC of Physica Activity for Health: a consensus statement from the British Association of Sport and Exercise Sciences. J Sports Sci. 2010; 28(6):573-91.

25. Haskell WL, Lee IM, Pate RR, Powell KE, Blair SN, Franklin BA, et al. Physical activity and public health: updated recommendation for adults from the AmericanCollege of Sports Medicine and the American Heart Association. Circulation. 2007;116(9):1081-93. 